\title{
EXOESQUELETO ACTUADO POR SMA PARA MOVILIZACIÓN DE LA MUÑECA
}

\author{
D. Copaci, D. Blanco, I. Guerra \\ Universidad Carlos III de Madrid, Avda. de la Universidad 30, Leganés-Madrid, dcopaci@ing.uc3m.es \\ S. Collado Vázquez, M. Pérez de Heredia \\ Universidad Rey Juan Carlos, Avda. Atenas, Alcorcón-Madrid, susana.collado@urjc.es
}

\section{Resumen}

La mano juega un papel fundamental en la relación del ser humano con su entorno, siendo las tareas de manipulación de enorme importancia en la vida diaria. Pero la mano es una extremidad frágil, un gran número de enfermedades y trastornos musculo-esqueléticos y neurológicos derivan en la pérdida total o parcial de la movilidad de esta extremidad. De aquí la importancia de las terapias de rehabilitación dirigidas a recuperar y/o mantener su movilidad. En este artículo se presenta el diseño preliminar de un dispositivo robótico para la rehabilitación de la muñeca con 2 g.d.l., con actuadores basados en fibras de SMA (Shape Memory Alloy). Gracias a este sistema de actuación el exoesqueleto es extremadamente ligero, de actuación silenciosa y con una estructura muy simple. El número de actuadores y el diseño preliminar se han calculado a partir de la simulación del comportamiento biomecánico del cuerpo humano con un tipo específico de pacientes.

Palabras clave: robots asistenciales, robótica médica, rehabilitación, exoesqueletos, actuadores avanzados.

\section{INTRODUCCIÓN}

La capacidad para realizar tareas de manipulación es fundamental para el ser humano. Son muchas las actividades de la vida diaria que requieren de la movilización de la mano y de la articulación de la muñeca para su correcta ejecución. Sin embargo, un gran número de enfermedades y trastornos musculo-esqueléticos y neurológicos derivan en la pérdida de movilidad en la muñeca y esto conlleva una incapacitación total o parcial para realizar de forma autónoma tareas de manipulación cotidianas. La ingeniería de rehabilitación ha llevado a cabo un gran esfuerzo en los últimos años para desarrollar tecnologías que sirvan de ayuda en la recuperación de la movilidad y capacidad de manipulación y los avances en este área han sido muy notables. Entre las tecnologías más prometedoras se considera que las terapias robotizadas con exoesqueletos son muy beneficiosas para la re- habilitación de pacientes que requieren tratamientos repetitivos para la reeducación los movimientos perdidos [9] permitiendo reducir los costes de hospitalización.

En este sentido en los últimos años, se han desarrollado algunos sistemas para la movilización de la articulación de la muñeca, aunque prácticamente todos utilizan motores eléctricos o, en algunos casos, actuadores neumáticos. Entre los dispositivos comerciales con aplicación clínica para la movilización de la muñeca se pueden citar algunos robots tipo efector final (aquellos que entran en contacto con el paciente solo a través de una órtesis en el antebrazo o mano [10]) como el InMotion Wrist diseñado para desviaciones radial y cubital y flexo-extensión de la muñeca o el HWARD. Más relacionados con nuestra propuesta están el ArmeoSpring de Hocoma y el Supinator-Extender o el Hand-Mentor al tratarse de exoesqueletos que actúan sobre la flexo-extensión de la muñeca. Existen otros dispositivos, la mayoría de ellos en fase de investigación o prototipos, una amplia revisión de los cuales puede consultarse en [6] y [10]. En algunos casos los exoesqueletos citados, caso del ArmeoSpring cubren no solo la flexoextensión de la muñeca, también y la prensión manual. En otros casos, como el Hand-Mentor, el dispositivo cubre la flexoextensión de la muñeca a la vez que la movilizacion de los dedos. El sistema propuesto en este artículo se ha diseñado para la realización de ejercicios de rehabilitación de la muñeca exclusivamente. En un futuro se estudiará la posibilidad de combinar su actuación con un exoesqueleto para codo [1].

Nuestro objetivo con el trabajo que ahora se presenta es mejorar las prestaciones en cuanto a peso y confortabilidad respecto a los dispositivos existentes, manteniendo las características de rangos, velocidades y pares desarrollados que permitan una correcta ejecución de las terapias de rehabilitación de la muñeca. 


\section{ARTICULACIÓN DE LA MUÑECA}

La muñeca o carpo es la colección de huesos y estructuras de tejidos que conecta la mano al antebrazo. Este complejo conjunto de huesos, ligamentos, tendones y tejidos blandos es capaz de ofrecer un arco amplio de movimiento que aumenta la función de la mano y dedos, otorgándole además un considerable grado de estabilidad. Esta articulación juega un papel fundamental en la vida diaria debido a que sus funciones cinemáticas permiten la orientación de la mano con respeto al antebrazo, y las cinéticas permiten transmitir las cargas del antebrazo a la mano y viceversa. Todas las articulaciones de la extremidad superior tienen como objetivo posicionar la mano para que pueda realizar las tareas deseadas, especialmente, la muñeca parece ser la clave de la función de la mano. La estabilidad de la muñeca afecta la capacidad de los dedos para flexionarse y extenderse [8], [5].

Es una articulación compleja formada de varias articulaciones que hacen la conexiones entre el hueso radio con los huesos de metacárpales (radiocarpal), el hueso cubito con el espacio ulnocarpal (distal radioulnar) y la conexiones entre los primera y segunda fila de los huesos cárpales (midcarpal). La masa ósea carpo está compuesta por ocho huesos organizados en dos filas de cuatro: escafoides, semilunar, piramidal, psiforme, trapecio, trapecoide, hueso grande y hueso ganchoso (Figura 1).

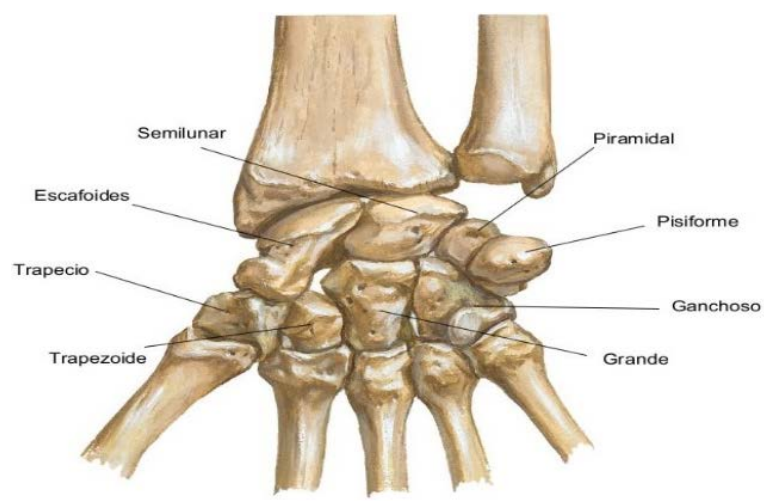

Figura 1: Articulación de la muñeca. Huesos del carpo, ulna y radio.

\section{DISEÑO MECÁNICO}

En este artículo se presenta el diseño preliminar de un exoesqueleto para la rehabilitación de la muñeca, tanto para el miembro derecho como para el izquierdo, con 2 g.d.l., actuado con fibras de
SMA, para un paciente tipo: hombre con $75 \mathrm{Kg}$. de peso y $1.75 \mathrm{~m}$. de altura.

\subsection{Simulación Biomecánica}

El uso de herramientas de simulación juega un papel fundamental en el desarrollo de cualquier dispositivo robótico. Hoy en día se considera imprescindible analizar el funcionamiento de los sistemas y las diversas soluciones de diseño propuestas antes de proceder a su fabricación de forma que muchas de las iteraciones del proceso de diseño puedan llevarse a cabo sin añadir costes de fabricación. En este trabajo se ha utilizado el software de simulación musculoesquelético "Biomechanics of Bodies" (BoB) [13] para estimar los pares necesarios en las articulaciones para un individuo específico. Esta herramienta software, construida como toolbox de Matlab/Simulink, es capaz de simular el comportamiento dinámico del cuerpo humano. Teniendo como entradas la altura, peso y movimiento a realizar por el individuo, se pueden obtener como datos de salida, entre otros, los pares de las articulaciones [2]. En nuestro caso, la simulación se ha configurado con los siguientes parámetros : peso $75 \mathrm{~kg}$., altura 1.75 $\mathrm{m}$ y trayectoria de la muñeca en flexo-extensión con 90 grados de flexión y 85 grados de extensión con una frecuencia de movimiento de $0.25 \mathrm{~Hz}$. La frecuencia del movimiento se ha considerado como máxima velocidad con cual se puede ejecutar la terapia de rehabilitación. El actuador en la parte de recuperación, en este caso coincidiendo con la extensión de la muñeca puede ser más lento. Como resultados de la simulación, para completar la tarea propuesta de rehabilitación de forma conveniente se requiere un par de $0.3 \mathrm{Nm}$ en la articulación de la muñeca (ver Figura 2). En el caso de desviación radial y desviación cubital, aunque la orientación de la mano puede cambiar, los pares de la articulación de la muñeca no pueden superar $0.3 \mathrm{Nm}$. En esta simulación se ha asumido el caso más desfavorable en el que el paciente tiene pérdida completa de la función motora de la articulación y toda la fuerza debe ser realizada por el exoesqueleto.

\subsection{Actuadores basados en SMA}

Los actuadores SMA tienen numerosas características interesantes, tales como su bajo peso o alta relación fuerza-peso, que las hacen una alternativa potencial a las tecnologías de actuación tradicionales en campos tales como aplicaciones espaciales, dispositivos quirúrgicos o robots "vestibles". En la publicación previa [12], se presentaron los primeros resultados que demuestran la viabilidad del actuador de SMA propuesto para 


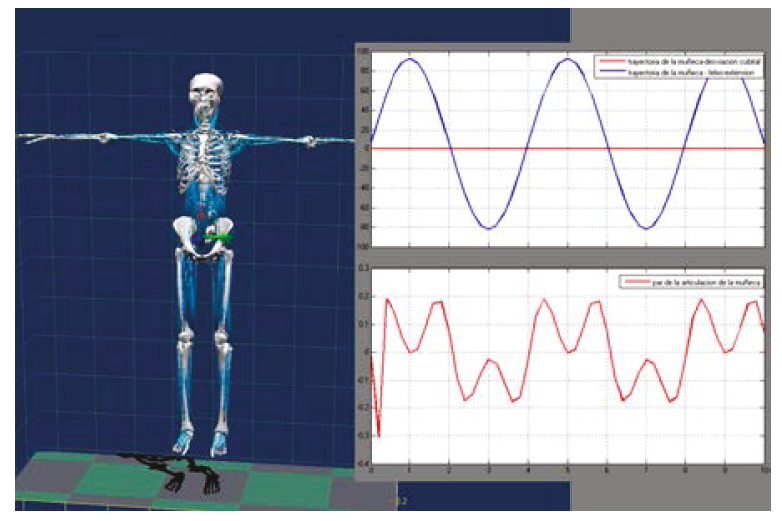

Figura 2: A la izquierda la interfaz del simulador BoB configurado para el movimiento de flexoextensión de la muñeca. A la derecha, trayectoria de entrada y valores de par necesario resultantes de la simulación.

su uso en robots portables.

Se ha diseñado un programa de simulación en Matlab/Simulink capaz de calcular el número y longitud de los actuadores SMA necesarios considerando el par necesario para la articulación de la muñeca y otros parámetros de diseño tales como: radio de las poleas usadas para transformar el movimiento de traslación en rotación, diámetro de la fibra de SMA, composición del SMA (NiTi y otras aleaciones). El esquema de simulación puede verse en la Figura 3.

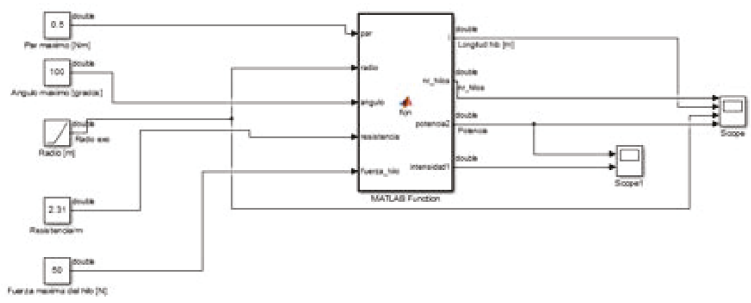

Figura 3: Programa de Matlab/Simulink para calcular las características óptimas del actuador.

Se ha determinado que la mejor aleación es SmartFlex® con un diámetro de $0.5 \mathrm{~mm}$. Además, las fibras de SMA se introducen en un cable Bowden que además de permitir fijar la estructura (uno de los extremos del SMA se crimpa en el extremo del Bowden) sirve para disipación del calor en la fase de enfriamiento. La elección del número de fibras se hizo teniendo en cuenta el peso adicional del exoesqueleto y un posible peso adicional en la mano, de aproximadamente $0.3 \mathrm{Kg}$. En este caso el par total de la articulación del exoesqueleto para la muñeca se ha considerado de aproximadamente de $0.5 \mathrm{Nm}$.

En función de estos resultados, para movilización de la muñeca se necesitan 1 hilo de SMA para cada g.d.l., con una longitud de aproximadamente $1 \mathrm{~m}$. Debido a la estructura del actuador, la longitud total de $1 \mathrm{~m}$ se puede adaptar o enrollar en función de la forma donde está emplazado, sin necesidad de estar recto.

En función de las características del hilo elegido, los tiempos más lentos de actuación se encuentran en la fase de recuperación del actuador (correspondiente al movimiento de extensión) de aproximadamente 10 segundos, el tiempo de enfriamiento dependiendo de la temperatura del ambiente. En esta aproximación se ha tenido en cuenta las posibles perdidas por fricción que pueden tener entre el hilo de SMA y el material de aislamiento eléctrico del cable Bowden.

\subsection{Diseño del exoesqueleto}

En función del análisis biomecánico y los actuadores seleccionados se ha propuesto un primer diseño del exoesqueleto que se muestra en la Figura 4. Toda la estructura del exoesqueleto se va emplazar sobre un guante que permite la sujeción con la mano humana. De esta manera el diseño propuesto permite una buena sujeción con la mano humana y minimiza el tiempo de colocación. Los puntos de sujeción con el cuerpo humano permiten transmitir de forma directa a la mano y el antebrazo las fuerzas generadas por el exoesqueleto. Por el intermedio del material del guante y el material de protección del exoesqueleto se amortiguan las presiones sobre la piel manteniendo una interacción confortable entre el dispositivo robótico y el cuerpo humano.

El exoesqueleto se ha diseñado con partes simples que facilitan el ensamblaje y que pueden ajustarse en función de las características antropométricas del paciente. Además se ha diseñado como un dispositivo de bajo coste cuyas partes pueden fabricarse con una impresora $3 \mathrm{D}$. Un primer prototipo impreso en plástico se puede ver en la Figura 6. El diseño del exoesqueleto muestra 2 g.d.l. uno para flexo-extensión y uno para la desviación cubital y radial. En los dos ejes el dispositivo robótico de rehabilitación dispone de muelles de torsión que ayudan a recuperar la forma inicial del actuador. Además el dispositivo está restringido mecánicamente a un cierto rango de movimiento 60 grados en flexión, 60 grados en extensión, 45 grados en desviación cubital y 20 grados desviación radial por la seguridad del paciente (Figura 5).

En cada eje de rotación el exoesqueleto presenta un sensor rotativo de posición (Bourns 3382G-1103G) que se va a utilizar para cerrar el bucle de control en movimiento y guardar una parte de los datos necesarios en el análisis de la evolución de 


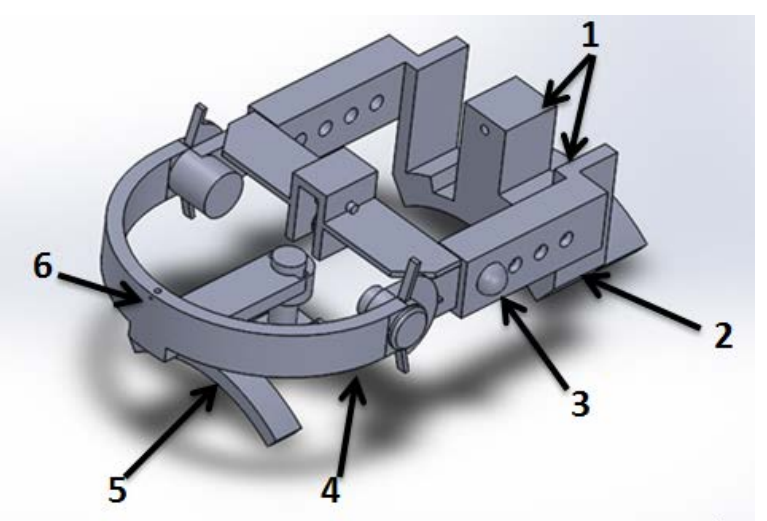

a)

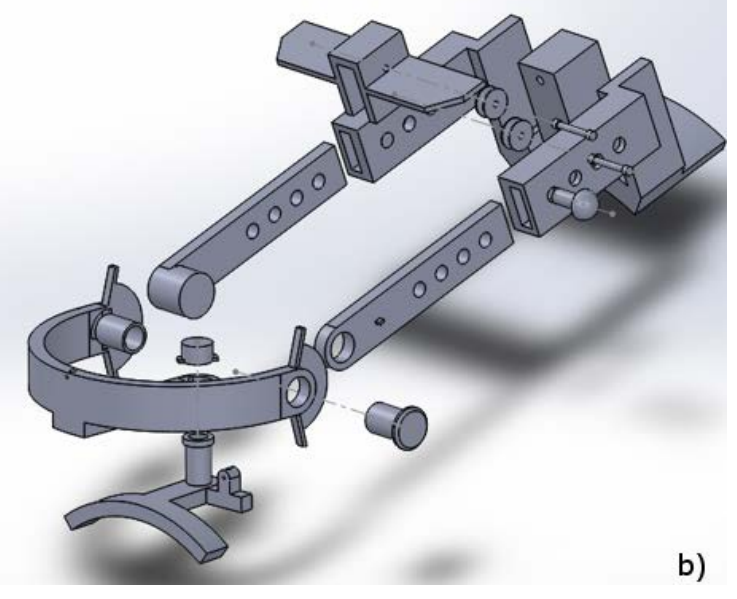

Figura 4: Exoesqueleto para la rehabilitación de la muñeca: a)1 -terminación del cable Bowden, 2 - punto fijo de sujeción con el antebrazo, 3 - sistema de ajuste en función del paciente, 4 - grado de libertad para flexo-extensión, 5 - grado de libertad para desviación cubital y desviación radial, 6 - punto de sujeción del actuador. b) Encaje de las piezas del primer prototipo.

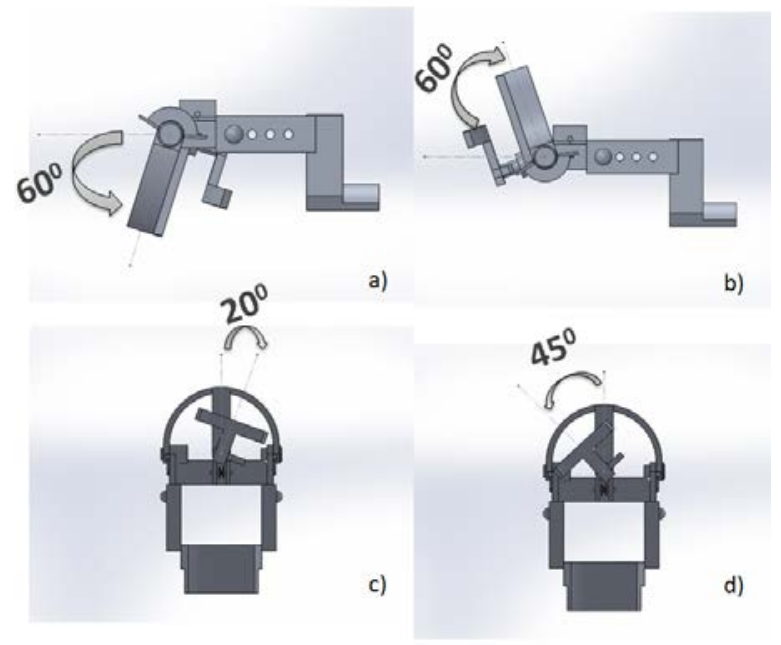

Figura 5: Movilización de la muñeca para la mano izquierda: a) rango de movimiento en flexión b) rango de movimiento en extensión c) rango de movimiento en desviación radial d) rango de movimiento en desviación cubital.

recuperación de los pacientes. En los puntos de sujeción de los actuadores con el exoesqueleto se ha situado una pieza de metal cilíndrica con un diámetro interno de $1 \mathrm{~mm}$ y dos prisioneros que atraviesan la pieza perpendicularmente. El hilo de SMA se introduce en la pieza y se va a sujetar con la ayuda de los dos prisioneros que presionan el hilo sobre la pared interna del cilindro.

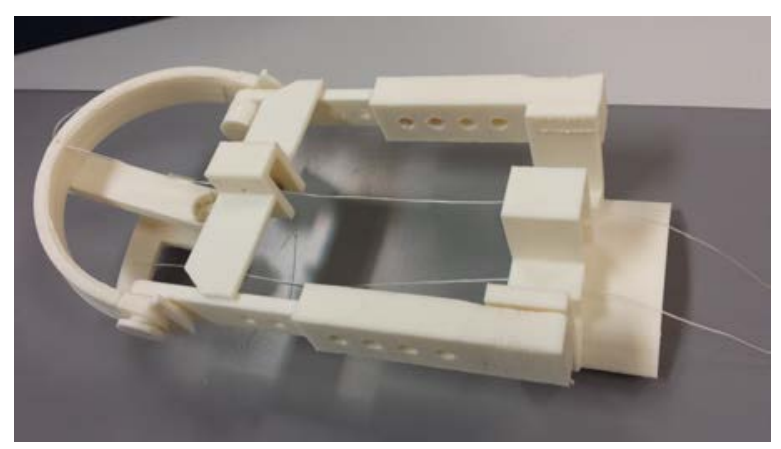

Figura 6: Prototipo del exoesqueleto para la muñeca impreso en plástico.

Este diseño presenta mejoras significativas respecto a los existentes en características como movilidad, confort, seguridad y facilidad de colocación. La ventaja principal viene dada por el uso de actuadores basados en fibras SMA, se trata de la drástica reducción del peso del dispositivo, apenas $0.15 \mathrm{Kg}$. Al mismo tiempo un muy bajo nivel de ruido durante la actuación debido a la ausencia de reductoras y motores en el mecanismo. La estructura del exoesqueleto se fijara a la mano y al antebrazo por el intermedio de un guante que 
además tiene como objetivo proteger el contacto entre el cuerpo humano y el dispositivo de rehabilitación.

\section{CONTROL}

En este capítulo se presenta la estrategia de control y la arquitectura hardware propuesta para el exoesqueleto para la movilización de la muñeca.

\subsection{Estrategia de control}

Una de las mayores dificultades que plantean los actuadores basados en fibras de SMA en lo relativo al diseño de los algoritmos de control, proviene de la no linealidad de su comportamiento debido a la histéresis térmica que aparece en la fase de transición [7].

Tomando como referencia el trabajo previo presentado en [12], se ha implementado un controlador bilinear de cuatro términos. Los controladores bilineales son una subserie de controladores no-lineales, se trata esencialmente de una combinación de un controlador lineal PID estándar con un compensador bilineal en cascada. Para este trabajo ha sido necesario un reajuste de los términos proporcional, integral, derivativo y bilineal en función de características del diseño.

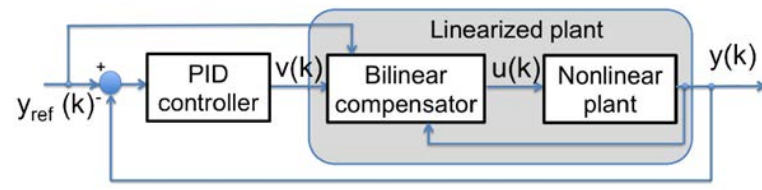

Figura 7: Control bilineal de 4 términos.

\subsection{Arquitectura hardware de control}

El hardware de control está basado en un NRF51822 Bluetooth con un microprocesador ARM@ Cortex $^{\mathrm{TM}}$ M0 CPU que, por sus prestaciones, peso y tamaño, resulta ideal para un dispositivo portable. La electrónica elegida puede ser programada desde Matlab/Simulink y se puede comunicar a traves de un puerto USBR [3]. La arquitectura hardware propuesta se basa en las normas IEC 61508, IEC 60601 y SIL 4 de acuerdo con los criterios de seguridad para los equipos médicos. Con la electrónica diseñada, esta arquitectura hardware de control es capaz de gestionar hasta 8 controladores para actuadores distintos. En el caso del exoesqueleto de la muñeca sólo son necesarios 2 controladores. Además la arquitectura hardware elegida está basada en una electrónica de bajo coste.

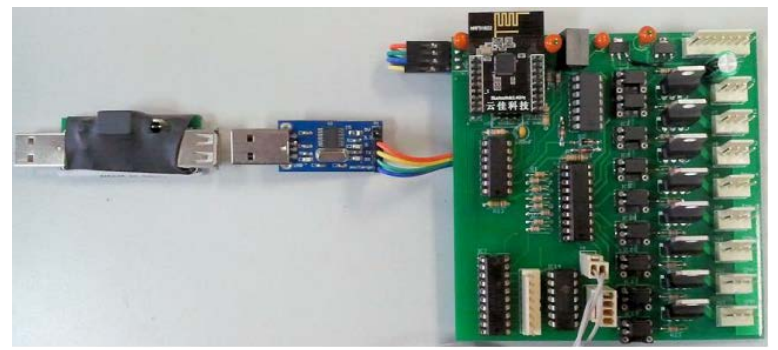

Figura 8: Arquitectura hardware de control.

\section{CONCLUSIONES}

Se ha presentado el diseño preliminar de un exoesqueleto actuado con SMA (sin motores) para la movilización de la muñeca. El dispositivo de actuación propuesto permite reducir drásticamente el peso del exoesqueleto a solo $0.15 \mathrm{Kg}$, se consigue además una actuación silenciosa lo que redunda en un incremento del confort del dispositivo. Se espera que ambas características favorezcan su aceptación por parte de los pacientes.

Este diseño preliminar se ha fabricado con una impresora 3D, en filamento de plástico ABS para poder analizar el diseño de forma conjunta con los sistemas de actuación. El dispositivo final va ser fabricado en una aleación y plástico.

Para el desarrollo del dispositivo de rehabilitación además de las características como: la seguridad de los pacientes, portabilidad del dispositivo, confort, eficiencia en las tareas de rehabilitación, un factor de diseño que se ha considerado importante lo ha constituido el coste del mismo, se ha pensado como un dispositivo de bajo coste que pueda ser adquirido por los hospitales especializados e incluso de los propios pacientes.

\section{Agradecimientos}

Los autores expresan su agradecimiento por los fondos recibidos a través de los proyectos RoboHealth (DPI2013-47944-C4-3-R) y RoboCity2030III-CM project (Robótica aplicada a la mejora de la cal-idad de vida de los ciudadanos. Fase III; S2013/MIT-2748), financiado por Programas de Actividades I $+D$ en la Comunidad de Madrid y cofinanciado por Fondos Estructurales de la EU.

\section{Referencias}

[1] Alguacil, I.M., Monge, E., Copaci, D-S., Blanco, D., Pérez De Heredia, M., Collado, S.."Diseño de un Exoesqueleto por Segmentos para Evaluación y Tratamiento del Miem- 
bro Superior". 54 Congreso Nacional de la Sociedad Española de Rehabilitación y Medicina Física (SERMEF). 2016.

[2] Copaci, D., Flores-Caballero, A., Blanco, D., Moreno, L.. "Herramienta de Simulación para el Desarrollo de Exoesqueletos basada en Matlab/Simulink", XXXV Jornadas de Automática. 2014.

[3] Flores, A., Copac,i D., Martín, A., Blanco, D., Moreno, L.. "Smooth and Accurate control of multiple Shape Memory Alloys based actuators via low cost embedded hardware". IEEE/RSJ Int. Conf. on Intelligent Robots and Systems. IROS 2012.

[4] Flores-Caballero, A.. "Sistema Avanzado de Prototipado Rápido para Control en Exoesqueletos y dispositivos Mecatrónicos", Tesis Doctoral, Universidad Carlos III de Madrid. 2014.

[5] Knudson Duane, Fundamentals of Biomechanics, Second Edition, Department of Kinesiology, California State University at Chico, 2007.

[6] Maciejasz, P., Eschweiler, J., Gerlach-Hahn, K., Jansen-Troy, A.. and Leonhardt, S.. " A survey on robotic devices for upper limb rehabilitation" Journal of Neuro Engineering and Rehabilitation, 2014, 11:3.

[7] Martin Clemente, A.. "Modelado y control de sistemas no lineales de tipo SMA", Tesis Doctoral, Universidad Carlos III de Madrid. 2014.

[8] Nordin, M., and Frankel, V. H.. "Basic Biomechanics of the musculoskeletal system", Fourth, North American Edition 2001.

[9] Pons, L. J.. "Wearable Robots: Biomechatronic Exoskeletons", Wiley Editorial, John Wiley and Sons, Inc. 2008.

[10] Rodríguez-Prunotto, L. et al. "Terapia Robótica para la rehabilitación del miembro superior en patología neurológica". Rehabilitación (Madr). 2014; 48(2): 104-128.

[11] Villoslada, A., Flores-Caballero, A., Copaci, D., Blanco, D., Moreno, L.. "High displacement flexible Shape Memory Alloy actuator for soft wearable robots", Robots and Autonomous Systems: Special Issue on Wearable Robotics for Motion Assistance and Rehabilitation 2014.

[12] Villoslada, A., Escudero,N., Flores, A., Martin, F., Rivera,C., Moreno, L., Collado,M..
"Position control of a shape memory alloy actuator using a four-term bilinear PID controller", SENSORS AND ACTUATORS A PHYSICAL 236, Oct. 2015.

[13] Wagner, D.W., Stepanyan, V., Shippen, J.M., DeMers, M.S., Gibbons, R.S., Andrews, B.J., Creasey, G.H., and Beaupre, G.S.. "Consistency Among Musculoskeletal Models: Caveat Utilitor". Annals of Biomedical Engineering 41 (8), 1787-1799. Available from http://dx.doi.org/10.1007/s10439013-0843-1. 2013. 